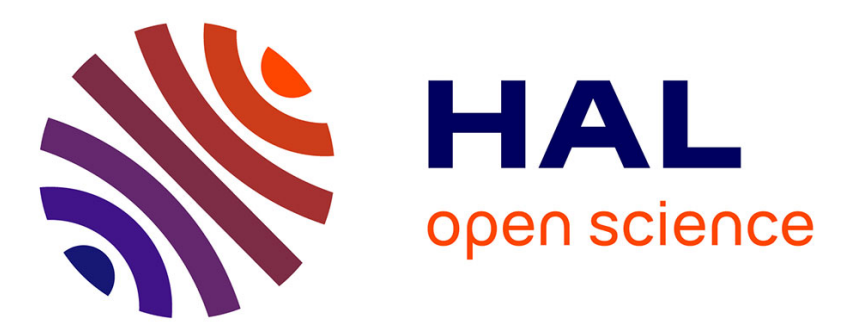

\title{
Detection of localized structures from multispacecraft data: Adaptive correlation function
}

\author{
L. Rezeau, G. Belmont, F. Reberac
}

\section{To cite this version:}

L. Rezeau, G. Belmont, F. Reberac. Detection of localized structures from multispacecraft data: Adaptive correlation function. Journal of Geophysical Research, 1998, 103, pp.2319-2326. 10.1029/97JA02666 . hal-00408538

\section{HAL Id: hal-00408538 \\ https://hal.science/hal-00408538}

Submitted on 13 Mar 2021

HAL is a multi-disciplinary open access archive for the deposit and dissemination of scientific research documents, whether they are published or not. The documents may come from teaching and research institutions in France or abroad, or from public or private research centers.
L'archive ouverte pluridisciplinaire HAL, est destinée au dépôt et à la diffusion de documents scientifiques de niveau recherche, publiés ou non, émanant des établissements d'enseignement et de recherche français ou étrangers, des laboratoires publics ou privés. 


\title{
Detection of localized structures from multispacecraft data: Adaptive correlation function
}

\author{
L. Rezeau, G. Belmont, and F. Reberac \\ Centre d'étude des Evironnements Terrestre et Planétaires, Université de Versailles Saint-Quentin, Vélizy, France
}

\begin{abstract}
When several spacecraft pass at different distances from a localized structure like a fieldaligned current tube, the corresponding magnetic signatures recorded on each spacecraft generally show different timescales, due to the different "viewpoints" of the spacecraft. For this reason, it is impossible to identify the presence of a unique structure from these signatures by using the classical correlation functions. In this paper, we present an "adaptive correlation function" which is a new analysis tool allowing to look for such "correlations" whatever the dilation factor is between two signals.
\end{abstract}

\section{Introduction}

In space plasmas, many objects of physical significance are localized in space, and their signatures are thus concentrated in time along the spacecraft trajectories. A paradigm for such structures can be a static cylindrical fieldaligned current tube (FAC), resulting for instance from the filamentation of a current sheet. As the magnetic field created by a FAC is easy to calculate, it is possible to search the magnetic field data for resembling signatures [Robert et al., 1984]; when such a signature is visually picked out, one can determine the few parameters of the model by a numerical fit (intensity of the current, distance from the spacecraft, and relative velocity). The short large amplitude magnetic structures (SLAMS), [Schwartz et al., 1992; Dudok de Wit and Krasnosel'skikh, 1995], which are observed upstream of quasi-parallel shocks, are other structures (more complex but still quasi-static) which can also be investigated in the same spirit.

When trying to extend the above method, one comes up against two limitations:

1. There is no uniqueness of the interpretation and most generally no means for testing the validity of the model chosen for the observed signature. This first limitation is met in particular when the structure can be supposed to have a temporal evolution, as in the case of kinetic Alfven waves [Chmyrev et al., 1988, Rezeau et al., 1993]: the eigenfrequency then mixes with the Doppler frequency because of the apparent motion and the number of independent data necessary to disentangle time and space variations becomes much larger. It is quite possible that the same event identified as a FAC from the only magnetic field data at one spacecraft can be interpreted as well by a kinetic Alfven wave or by a combination of both (Alfven solitons). The only way to go beyond this limitation is to increase the amount of data comparable with the model, so decreasing the probability of multiple interpretations (in particular by the use of multispacecraft experiments).

Copyright 1998 by the American Geophysical Union.

Paper number 97JA02666.

0148-0227/98/97JA-02666\$09.00
2. Even when localized signatures can be picked out visually in different signals, the question of whether these signatures are due to the same structure or have independent causes cannot always be answered. In particular, the use of correlation methods often fails when the signals are recorded on different spacecraft. We will try to bring a solution to this specific problem.

In order to restrict the possibilities of multiple interpretations, especially to disentangle space and time variations, the best way thus appears to be the use of simultaneous data from several close spacecraft. This was one of the motivations of multispacecraft missions such as ISEE or Interball, and a lot of work has been done in this field in the frame of the CLUSTER project preparation. The data processing methods now exist to get decisive physical information out of the data when the measurements are made inside a structure with a typical scale larger than the interspacecraft distance, e.g., intensity of the local current imbedded [Robert and Roux, 1990] or a study of waves through an estimation of thewavenumber spectrum for each frequency [Pinçon and Lefeuvre, 1991] or through recognition of the wave mode [Motschmann and Glassmeier, 1995]. Nevertheless, as discussed by Rezeau et al. [1990], when the spacecraft remain outside of a small current structure (compared to interspacecraft distance), the magnetic signatures recorded on each of them present different characteristic scales and amplitudes because of the different viewpoints of the different spacecraft: as the magnetic field created by a current tube decreases with the distance to the source, a spacecraft passing by the tube at a short distance records a narrow and intense signature, whereas a spacecraft passing at a large distance records a wide signature with a weak intensity. Then, all classical tools such as correlation functions or spectral analysis fail in diagnosing the structure even in this simple case of a pure FAC. The same conclusions came out of an experimental study performed on ISEE 1 and 2 data [Rezeau et al., 1993] in a case where the observations are very similar on both spacecraft and where many indications show that it is most probable that the same localized structure is observed on both spacecraft: the spectral analysis indicates a frequency shift of the peaks in the power spectra, and consequently the cross-correlation functions between the two 
spacecraft signals have a very weak maximum. These studies indicate that a satisfactory method should take into account this viewpoint effect which is different from one spacecraft to another. A new method has been developed, the adaptive correlation function, which is derived from the wavelet analysis of signals. The application of the method to ISEE data will show its efficiency. It must be noticed that the present work is limited to the identification of signatures of different scales but similar shapes. It could be extended in the future to signals that are physically related (due for instance to the derivatives contained in the Maxwell equations) without having the same shape.

The method is obviously not limited to the particular case presented here of magnetic signatures, it should be useful in any case when geometrical effects around a localized structure are to be taken into account whatever the data. It could be applied to the density or the velocity of the plasma.

\section{Data and Method Baseline}

The method will be described and applied to the same case as in the work of Rezeau et al. [1993]: a localized structure observed on magnetic fluctuations at the inner edge of the boundary layer, with a small delay between ISEE 1 and 2 (November 8, 1977, around 0255 UT). The data are the flux gate magnetometers data since the ISEE search-coil magnetometers frequency range does not allow the exploration of the ultra-low-frequency range (Figure 1). Nevertheless, a filtering by subtraction of a running average over $5 \mathrm{~s}$ has been applied to eliminate the large-scale

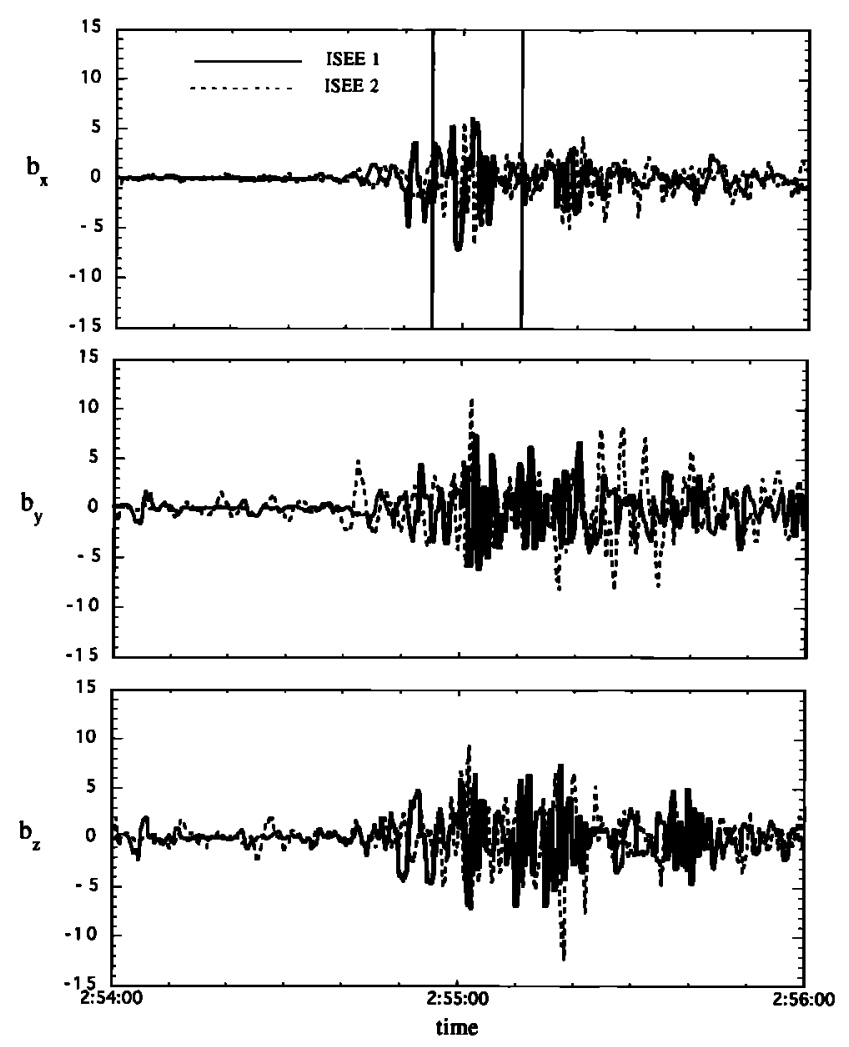

Figure 1. Waveforms of the magnetic fluctuations (nanoteslas) observed on ISEE 1 and 2, projected in the magnetopause $\mathrm{LMN}$ frame. variations of the field (which are similar on both spacecraft) and keep only the fluctuations (which could be different from one spacecraft to another). It makes the inspection of the fluctuations clearer, but it is not a necessary step; the method presented here could be applied on the nonfiltered data. The results obtained by Rezeau et al. [1993] show that the signals on the two spacecraft are correlated (the maximum correlation $(0.6)$ is obtained for a delay equal to $2 \mathrm{~s}$ ) but that there is a discrepancy in the spectral features (the relative maximum of the spectrum is at $0.6 \mathrm{~Hz}$ on ISEE 1 and $0.8 \mathrm{~Hz}$ on ISEE 2 . Nevertheless, they are likely to be interpreted as two signatures of a unique structure convected by the plasma flow.

The baseline of the adaptive correlation method is derived from the wavelet analysis, but it is not a new wavelet theory. The main properties of the wavelet transform with respect to the Fourier transform are the localisation of the analyzing function and the self-similarity. For instance, if we consider the Morlet wavelet analysis, it can be compared to the Fourier transform: the elementary analyzing function is a limited complex exponential instead of an unlimited complex exponential [Grossmann et al., 1989; Lagoutte et al., 1992]. The limitation is obtained by multiplication by a gaussian function with a width equal to a few periods of the complex exponential whatever the frequency. For this reason, the wavelet analysis is better adapted to the study of transient or localized signals than the Fourier transform. When applied to the signals of Figure 1, a wavelet analysis will emphasize the fluctuations around 0255:00 UT on both spacecraft; it will show that the maxima are observed at times slightly different with close timescales, but it will obviously not give any indication on their possible correlation. The adaptive correlation function is a correlation function modified to get a localized diagnosis in the same spirit as in wavelet theory. The method consists in the following steps: (1) pick out an event on one component recorded on one of the two spacecraft (the corresponding data will be referred hereafter as RS, reference signal), (2) extract the desired pattern (supposed to be related to an interesting physical phenomenon) from it by filtering to prepare a reference function (similar to mother wavelet in wavelet analysis), (3) dilate (or contract) and delay this function to analyse another signal (AS, analyzed signal), generally the same component recorded on the other spacecraft. The result of this analysis will be a threedimensional diagram similar to the wavelet scalogram, with time as horizontal axis and scale (i.e. dilation relative to the RS scale) as vertical axis. It will evidence the times when signals are correlated on the two spacecraft, and the relative time scales of the two viewpoints. From this comes the name adaptive correlation given to the method.

\section{Description of the Method}

The first step is the choice of the RS from the visual identification of an "event." On the example of Figure 1, we select about $10 \mathrm{~s}$ around 0255:00 UT on the $B_{\mathcal{X}}$ component recorded on ISEE 2 . The simplest way would then consist in taking the signal itself as analyzing wavelet. The tests that have been performed show that, because of the superimposed noise, this method is not efficient (see section 4). The signal has to be filtered before use, and the method chosen here is the singular-spectrum analysis (SSA). As it will be shown below, this method is very efficient to separate trend and oscillatory part of the signal, without fixing an arbitrary low- 
frequency cutoff. The software used is the one developed by Dettinger et al. [1995] and described by Vautard et al. [1992]. This spectral analysis method is dedicated to short and noisy signals. It is a decomposition of the signal on eigenelements, and the specificity of the method is that these eigenelements are data-adaptive; they are not given as inputs like in Fourier transform. After this decomposition on the eigenvectors, called principal components, the signal can be filtered by reconstructing it from only a subset of the principal components. On Figure 2, the method has been applied on the RS over $16 \mathrm{~s}$ beginning at 0254:54 UT. The original signal is displayed in Figure 2a (the running average filtering has not been applied before SSA analysis to bring out the capability of the method); in Figures $2 b-2 c$, the reconstruction of the signal on different subsets of principal components are shown. It evidences the efficiency of the method for separating the trend (low-frequency part of the signal), the oscillatory part and the high-frequency noise of the signal. The number of principal components on which the signal is split is a free parameter called the "embedding dimension"; it has been chosen here equal to one fourth of the total number of points of the time series. From the previous study [Rezeau et al., 1993], it seems that a localized structure is the cause for the oscillatory part of the signal around 0255 UT; we therefore choose the reconstruction on principal components number 3 and 4 as analyzing function.

Once the reference function $g$ is chosen, we use it to analyse a signal. For that purpose, we compute a scalogram, which is similar to a spectrogram but with a computation window increasing with scale in the same way as in wavelet analysis [Grossmann et al., 1989]. A family of daughter functions is generated by dilation and translation in time: $\mathrm{g}_{\mathrm{a}, \mathrm{b}}(\mathrm{t})$ (see Appendix for details). Both dilation and translation parameters are allowed to vary and sampled. The wavelet is translated with steps proportional to the width of the wavelet, in order to increase the time resolution when the scale decreases. For each value of $a$ and $b$ the correlation coefficient $c_{g, s}(a, b)$ is computed and displayed with a color code on the scalogram.

The result is shown in Figure $3 b$. The horizontal axis is time, the vertical axis is scale (or "dilation": scale is 1 for the mother wavelet); the maximum scale is at the top of the diagram. The pixel width increases with scale, it is equal to $5 \%$ of the wavelet width. As a test, the program has been first applied to RS. As expected the maximum of the correlation function is obtained for a dilation equal to 1 , at time $t=0255: 01.6$, time of the maximum of the signal.

More interesting is the result shown on Plate $1 \mathrm{~b}$, where AS is the $B_{X}$ component recorded on ISEE 1 (Plate 1a). Four main maxima are seen with the characteristics shown in Table 1 (in order of decreasing value of the maximum). The first point is that none of these maxima correspond to a dilation equal to 1 , which means that the viewpoints of the two spacecraft are actually different. One of the maxima (3) evidences the same delay as the classical correlation computation (delay equal to $2 \mathrm{~s}$, [Rezeau et al., 1993]), it is obtained for a dilation equal to 1.3. This result is consistent with the spectral analysis performed in the same study, which gave frequency maxima at 0.6 and $0.8 \mathrm{~Hz}$, which means a scale factor of $0.8 / 0.6=1.33$ between the observations on the two spacecraft.

Plate Ic shows the final form proposed for the visualisation of the result. This last change aims to answer
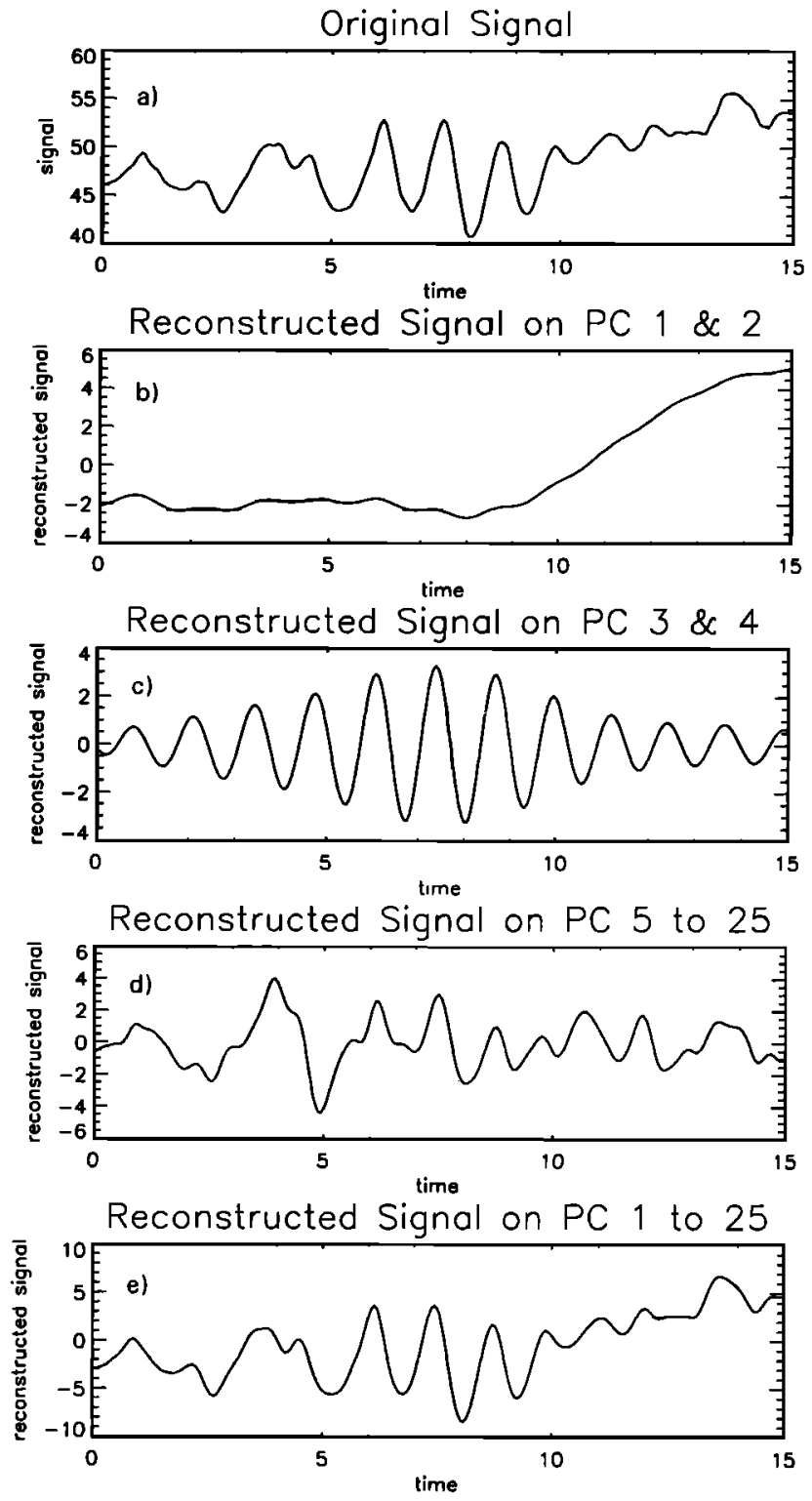

Figure 2. Singular-spectrum analysis (SSA) filtering. (a) Waveform of the reference signal ( $B_{x}$ component measured by ISEE 2, total magnetic field). The horizontal axis is delay in seconds from the beginning of the selection (0254:54). The time period presented corresponds to the vertical lines on Figure 1 (top). (b)-(e) Reconstruction of the signal using different subsets of principal components determined by SSA. Figure $2 e$ shows the reconstruction on the 25 first components: it is identical to the original signal (Figure 2a), except for the average which is lost by SSA.

the following difficulty: as the correlation coefficient has been calculated to be independent upon the power in the correlated signals, large values may correspond to very weak components in the analyzed signal. This property obviously increases the probability of spurious correlations between the analyzing wavelet and many small components of the signal which are not physically significant. To avoid cluttering the display with such parasitic correlations, Plate $1 \mathrm{~b}$ has been modified in the following way: instead of plotting the correlation coefficient, we only use it as a criterion to select the nonnormalized correlation function which is proportional 
a)

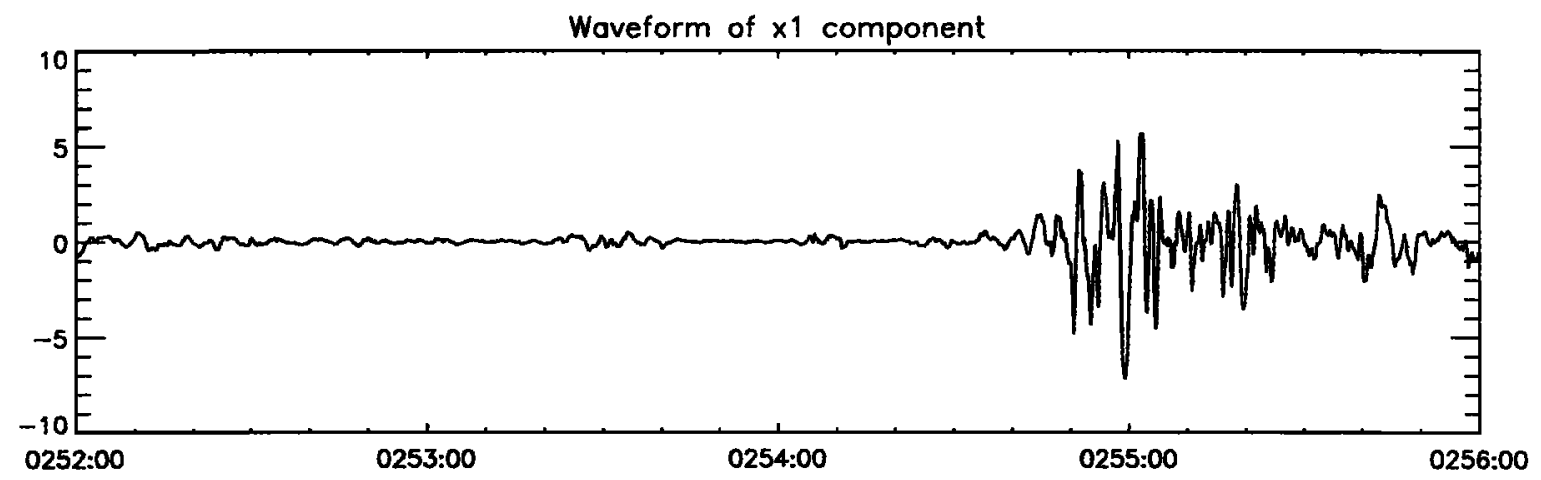

b)

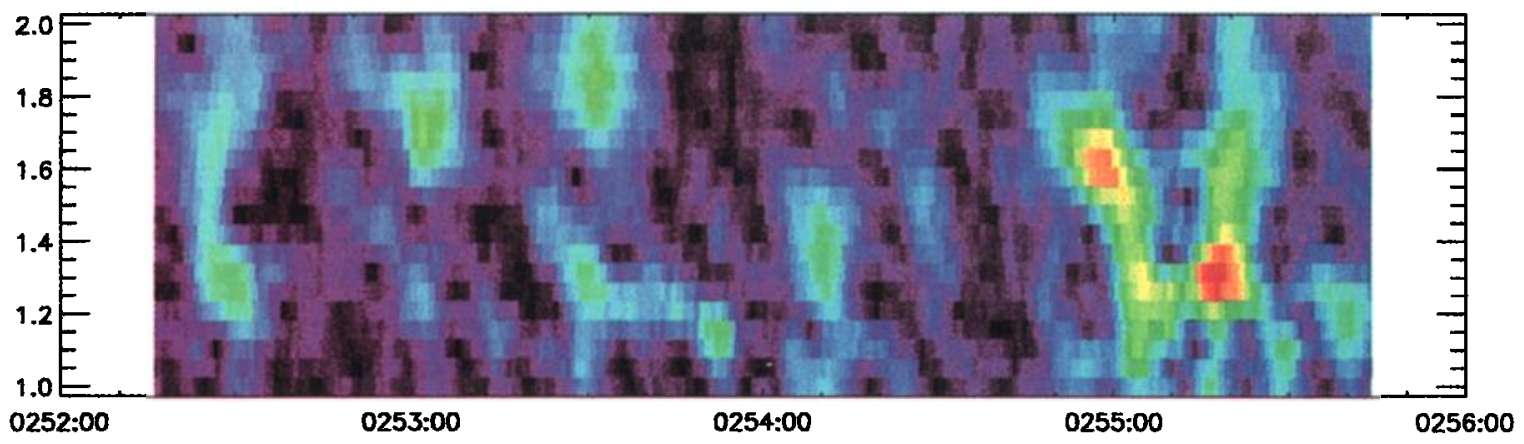

$\operatorname{mb}$ val $=5.530-03-\max$ val. $=6.730-01$

c)

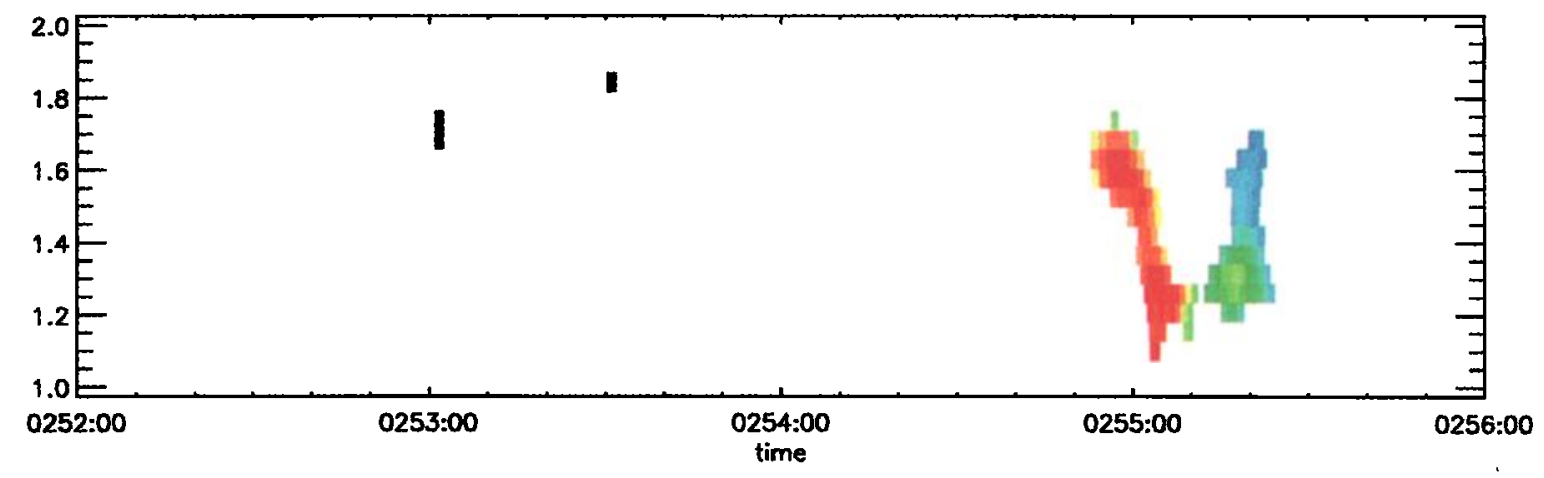

$\min \mathrm{val} .=1.300-03-\max \mathrm{val} .=6.81 \theta-02$

Plate 1. (a and b): Result of the analysis when applied to the same component on the other spacecraft (ISEE 1). The dilation scale goes from 1 to 2 . Four maxima are observed, they are labeled from 1 to 4 with decreasing correlation. (c) The normalized correlation is used as a filter for the plot of the nonnormalised correlation. The colored region is the region where the correlation is higher than 0.4 ; inside this region the color code goes from black to red with the amplitude of the signal.

to the intensity of the correlated signals. In the regions of the plane where the correlation coefficient is lower than a given threshold (chosen equal to 0.4 here), the color plot of the nonnormalized correlation function is masked. When it is plotted, this nonnormalized correlation is directly proportional to the amplitude of AS since the analyzing wavelet is normalized. The interpretation of the four maxima observed on Plate 1b is modified by this analysis, two of them (2 and 3) appear more interesting since they are both correlated and intense events (they are identified by $*$ in Table 1 and their intensities are respectively 0.0679 and 0.0681 ). Therefore, in addition to the maximum at 1.3 dilation (3), the maximum corresponding to 1.6 dilation (2) appears significant although it was not evidenced by the classical correlation analysis, the other one was the already identified one. The result of this analysis is that the signature observed on ISEE 2 appears to be correlated with two signatures on ISEE 1. This is still to be physically interpreted, but this example shows that the improvement of the correlation method was necessary.

\section{Discussion and Conclusion}

We have shown in this paper that the classical correlation methods used to detect in different signals similar wave forms with equal timescales cannot be used without change to detect 

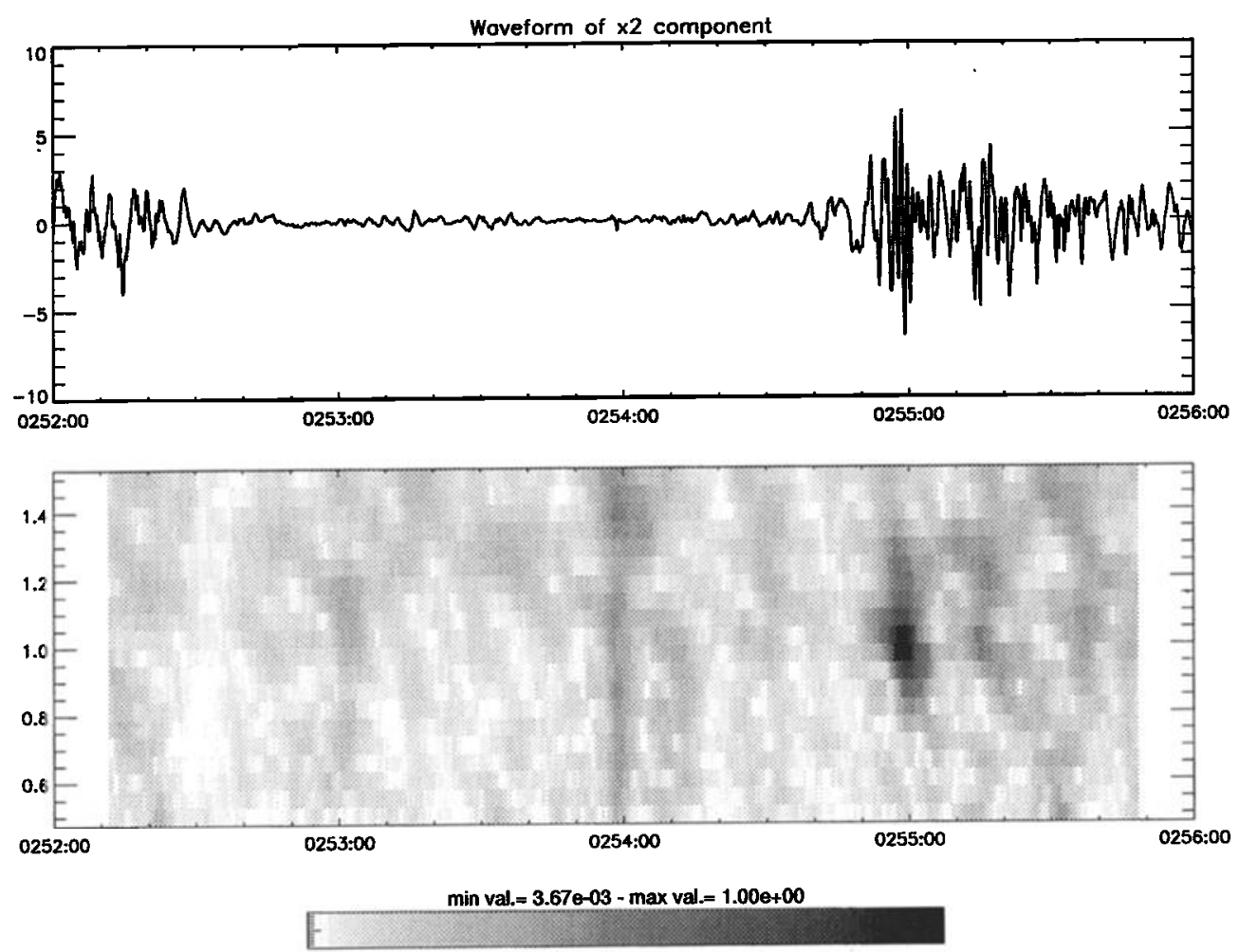

Figure 3. Result of the analysis when applied to the reference signal (Figure $3 \mathrm{~b}$ ). The horizontal axis is time. The dilation is shown on the vertical axis, with a linear scale from 0.5 to 1.5 . As expected when analyzing the reference signal, the maximum is obtained for a dilation equal to 1 .

the signatures of a spatial structure from different spacecraft, because of the different time scales implied by the different viewpoints of the spacecraft. The method proposed makes use of a filtering of one of the signals by singular spectrum analysis (to isolate the signature to be correlated) followed by a "wavelet correlation" with the second signal. Associated with an adequate visualisation, the method has been shown on an example to be powerful and to allow the detection of a correlation between two signals with a scale ratio different from 1 .

An element of the method has still to be discussed: why not to use the signal itself as an analyzing wavelet? It would seem easier than performing the SSA filtering before the analysis. The reason is that like the analyzed signal, the original signal contains many components, including highfrequency noise (and eventually trend). For the same reason as in the preceding section, keeping all of these components

Table 1. Characteristics of the Maxima Observed in Plate 1b (labeled from 1 to 4 With Decreasing Value of the Maximum)

\begin{tabular}{lcccc} 
Maxima & Time & Delay (s) & Dilation & Correlation \\
\hline 1 & $0255: 17.6$ & 16 & 1.32 & 0.68 \\
$2^{*}$ & $0254: 58.8$ & -2.8 & 1.58 & 0.57 \\
$3^{*}$ & $0255: 03.9$ & 2.3 & 1.32 & 0.49 \\
4 & $0255: 19.0$ & 17.4 & 1.58 & 0.48 \\
\hline
\end{tabular}

* events for which the nonnormalized correlation is the most intense (see Plate1c) greatly increases the number of spurious correlations between the analysed signal and many small components of the original signal. When doing so, the result is thus again a figure cluttered by a lot of nonsignificant high values of the correlation coefficient, varying substantially from one wavelet translation step to the next because of the highfrequency noise; the method would then demand a step as small as possible (sample time) because of these artificial fine structures.

With the filtered signal, on the contrary, a step corresponding to a fraction of the length of the analyzing function is sufficient (4\%). For a dilation equal to 1 , in our case, this means steps of 12 sample times and the obvious consequence is a significant save in computation time. It must be noted that larger pixels still give the same result, and we could have saved even more computation time.

From the results obtained for the given example, it appears that the interpretation is still not straightforward since the selected signature on ISEE 2 is correlated with two signatures on ISEE 1. Of course, we cannot grant that when two signatures are correlated, they do correspond to the same event. It is possible that one signature corresponds to the same event but that the other one is the signature of an event that is similar without being the same. It is also possible that the second event is seen by only one spacecraft and not by the other. This could lead to conclude that the mother function is so generic that it correlates with any wave packet: this is not true since only two events are selected by the method, while a visual inspection of the signals after 0255:00 UT evidences the existence of other wave packets that are not correlated with the mother function. However, the results 
obtained confirm that the signal processing tool that we propose is able not only to quantify correlations which are apparent by visual inspection but also to detect possible correlations which are less obvious at first glance.

The adaptive correlation function, in its present form, enables one to detect and identify the signatures that are due to the same localized structure when they are observed from the data of two different spacecraft, under the condition that the viewpoint effect does not modify the shape of the signature. This method appears as a necessary step before further developments, consisting in particular to directly correlate the data of more numerous spacecraft as in the CLUSTER project. As shown in the preceding study [Rezeau et al., 1993], with two spacecraft the position of the structure can be derived assuming it is stationary. The data from a third spacecraft would either confirm this position, either evidence a temporal evolution of the structure. Three spacecraft limit anyway this interpretation to a plane, a fourth one is necessary to get the information in all directions (for instance, a kinetic Alfven wave might have a parallel propagation together with a perpendicular evolution, convection or expansion).

One of the referees has pointed out that the problem we are trying to solve here has some similarities with a radar problem: the echo of the emitted, and perfectly known, radar pulse is to be recognized in the return signal in spite of the Doppler effect induced by the motion of the target. Techniques have therefore been develóped by radar scientists that are related to our adaptive correlation function, in particular the ambiguity function [Rihaczek, 1960]. Some authors [Bertrand et al., 1994, 1995] suggest that this function can be computed using the Mellin transform; even if the problem is not exactly the same in our case (the input signal is not controllable), the use of similar algorithms might also induce a save in computation time.

\section{Appendix}

Let $x(t)$ be the reference signal (RS), on which a localized structure has been identified by visual inspection. This observed signature is first isolated by picking up a portion of the original signal in a window $\left[t_{o}-\delta t, t_{o}+\delta t\right]$. The position and the width of this window are chosen in order to include the whole structure. The mother analyzing function is obtained from SSA filtering (using the freeware program developed by Dettinger et al. [1995]): $g=K R C_{p-q}[x]$, where $K$ is a normalization constant and $R C_{p-q}[x]$ is the reconstructed signal on principal components $p$ to $q$. Both $p$ and $q$ are chosen in order to reproduce the gross features of the observed structure and, as long as long as the structure looks like a wave packet, the value of $g$ is generally small on the edges of the window. Nevertheless, to make sure that no discontinuity pollutes the correlation calculation, a taper (smoothing of the edges by a square cosine, applied to the first and last $10 \%$ of the window) is applied to the edge points of the mother function.

From this mother function $g$, daughter functions $g_{a, b}$ are deduced: $\quad g_{a, b}(t)=K_{a} g[(t-b) / a]$ where $K_{a}$ is another normalisation constant, $b$ is a delay and $a$ the dilation. These daughter functions are used to compute the scalogram of a given signal $s(t)$ :

$$
C_{g, s}(a, b)=\int_{b-a \delta t}^{b+a \delta t} g_{a, b}(t) s(t) d t,
$$

where the integration is limited to the width of the $g$ function. $C_{g, s}$ is proportional to the amplitudes of the signal $s(t)$ and of the analyzing function; in order to calculate a normalized correlation function we define

$$
c_{g, s}(a, b)=C_{g, s}(a, b) / \sqrt{E\left(g_{a, b}\right) E(s)},
$$

where $E(s)$ and $E\left(g_{a, b}\right)$ are energies contained in $s$ and $g_{a, b}$ :

$$
E(f)=\int_{b-a \delta t}^{b+a \delta t} f^{2}(t) d t
$$

is the energy. With this definition, $c_{g, s}$ is smaller than 1 .

With regard to calculation of the normalization coefficients $K$ and $K_{a}$, as in the wavelet theory, it is necessary to have $K_{a}=1 / \sqrt{a}$, to ensure the conservation of energy whatever the dilation of the function. To compute $K$, the reference signal itself is analyzed; the maximum

$$
c_{g, x}\left(1, b_{0}\right)=K /{\sqrt{E\left(g_{1, b_{0}}\right) E(x)}}^{b_{0}+\delta t} \int_{b_{0}-\delta t} R C_{3-4}[x](t) x(t) d t
$$

is obtained for $a=1$ and a time $b_{0}$. This time $b_{0}$ is exactly the time $t_{b}$ where the mother function has been extracted from the original signal. $K$ is chosen to set $c_{g, x}\left(1, b_{0}\right)=1$.

Acknowledgments: The authors wish to thank C. T. Russell (UCLA) for having kindly provided them with the ISEE magnetometer data. They also acknowledge the careful reading of the manuscript by $\mathrm{N}$. Cornilleau-Wehrlin and her useful comments.

The Editor thanks Steven Schwartz and Thierry Dudok de Wit for their assistance in evaluating this paper.

\section{References}

Bertrand J., P. Bertrand, and J-P. Ovarlez, Frequency-directivity scanning in laboratory radar imaging, Int. J. Imag. Syst. and Technol., 5, 39-51, 1994.

Bertrand J., P. Bertrand, and J-P. Ovarlez, The Mellin transform, in The Transforms and Applications Handbook, edited by A. D. Poularikas, chap.12, CRC Press, Boca Raton, Fla., 1995.

Chmyrev, V. M., S. V. Bilichenko, O. A. Pokhotelov, V. A. Marchenko, V. I. Lazarev, A. V. Streltsov and L. Stenflo, Alfven vortices and related phenomena in the Ionosphere and the magnetosphere, Phys. Scr., 38, 841-854, 1988.

Dettinger, M. D., M. Ghil, C. M. Strong, W. Weibel, and P. You, Software expedites singular-spectrum analysis, Eos, Trans. AGU, $76(2), 12,14,21,1995$

Dudok de Wit, T., and V.V. Krasnosel'skikh, Wavelet bicoherence analysis of strong plasma turbulence at the Earth's quasi-parallel bow shock, Phys. Plasmas, 2, 4307-4311, 1995.

Grossmann, A, R. Kronland-Martinet, and J. Morlet, Reading and understanding continuous wavelet transform, in Wavelets, TimeFrequency Methods and Phase Space, edited by J. M. Combes, A. Grossmann, and P. Tchamitchian, pp. 2-20 Springer-Verlag, New York, 1989.

Lagoutte, D., J. C. Cerisier, J. L. Plagnaud, J. P. Villain, and B. Forget, High latitude ionospheric electrostatic turbulence studied by means of the wavelet transform, J. Atmos. Terr. Phys., 54, 1283-1293, 1992.

Motschmann, U., and K.-H. Glassmeier, Mode recognition of MHD wave fields, Eur. Space Agency Spec. Publ., ESA SP-371, 103-106, 1995.

Pinçon, J.L., and F. Lefeuvre, Local characterisation of homogeneous turbulence in space plasma from simultaneous measurements of fields components at several points in space, J. Geophys. Res., 96, 1789-1802, 1991.

Rezeau, L., A. Roux, and C. T. Russell, Characterisation of small scale structures at the magnetopause from ISEE measurements, $J$. Geophys. Res., 98, 179-186, 1993. 
Rezeau, L., A. Roux, and N. Comilleau-Wehrlin, Multipoint study of small scale structures at the magnetopause, Eur. Space Agency Spec. Publ., ESA SP-306, 103-108, 1990.

Rihaczek, A. W., Principles of High Resolution Radar, McGraw-Hill, New York, 1969.

Robert, $\mathrm{P}$., and A. Roux, Accuracy of the estimate of $\mathrm{J}$ via multipoint measurements, Eur. Space Agency Spec. Publ., ESA SP-306, 29-35, 1990.

Robert, P., R. Gendrin, S. Perraut, and A. Roux, GEOS 2 identification of rapidly moving current structures in the equatorial outer magnetosphere during substorms, J. Geophys. Res., 89, 819-840, 1984.
Schwartz, S. J., D. Burgess, W. P. Wilkinson, R. L. Kessel, M. Dunlop, and $H$. Lühr, Observation of short large-amplitude magnetic structures at a quasi-parallel shock, J. Geophys. Res., 97, 4209, 1997

Vautard, R., P. Yiou, and M. Ghil, Singular-spectral analysis: A toolkit for short, noisy chaotic signals, Physica D, 58, 95-126, 1992.

G. Belmont, F. Reberac, and L. Rezeau, CETP/UVSQ, 10-12 avenue de l'Europe, 78140 Vélizy, France. (e-mail: rezeau@cetp.ipsl.fr)

(Received December 27, 1997; revised September 9, 1997; accepted September 14, 1997.) 